Editor's Note: These short, critical reviews of recent papers in the Journal, written exclusively by graduate students or postdoctoral fellows, are intended to summarize the important findings of the paper and provide additional insight and commentary. For more information on the format and purpose of the Journal Club, please see http://www.jneurosci.org/misc/ifa_features.shtml.

\title{
The Multiple Origins and Laminar Topography of the Hippocampal Theta Rhythm
}

\author{
Prasad Shirvalkar ${ }^{1}$ and Amir S. Bahar ${ }^{2}$ \\ ${ }^{1}$ Medical Scientist Training Program, Graduate School of Biological Sciences and ${ }^{2}$ Fishberg Department of Neuroscience, Mount Sinai School of Medicine, \\ New York, New York 10029 \\ Review of Montgomery et al. (http://www.jneurosci.org/cgi/content/full/29/5/1381)
}

The hippocampal theta rhythm (4-10 $\mathrm{Hz}$ ) is among the most widely studied brain rhythms. Theta rhythmic activity has been associated with cognitive performance in humans (Kahana, 2006) and spatial navigation and other memory tasks in rats (Huxter et al., 2008). Rhythmic activity in theta range reflects synchronous oscillations in the membrane currents of local dendrites and synapses (Buzsáki, 2002). Contemporary models of theta generation in the hippocampus posit that rhythmic input from the medial septum and other sources such as intrahippocampal recurrent collaterals and hippocampal voltage-gated ion channels are responsible (Bland and Oddie, 2001; Buzsáki, 2002). In this review, we discuss a recent study (Montgomery et al., 2009) that provides a compelling revision to these models and presents evidence for multiple local theta generators throughout the hippocampus. The authors report that theta oscillations varied between the different layers of the hippocampus and that these differences were, to a large extent, a result of local independent theta dipoles. In addition, they show that layer-

Received March 31, 2009; accepted April 20, 2009.

We thank Pamela Kennedy and Justin Riceberg for comments on this manuscript. A.S.B. is supported in part by a 2008 NARSAD Young Investigator Award.

Correspondence should beaddressed to AmirS. Bahar, Fishberg Department of Neuroscience, Mount Sinai School of Medicine, Box 1065, One Gustave L. Levy Place, New York, NY 10029. E-mail: amir.bahar@mssm.edu.

DOl:10.1523/JNEUROSCI.1528-09.2009

Copyright $\odot 2009$ Society for Neuroscience $\quad$ 0270-6474/09/297111-03\$15.00/0 specific modulation of theta may be linked to behavioral and cognitive operations.

Neural oscillations are typically detected from local field potential (LFP) recordings from the extracellular space. Waveform recordings of LFPs depend on conductivity (or resistivity) of the extracellular medium, local anatomy of neurites and synapses, and the electrode geometry in the extracellular space (Montgomery et al., 2009); interpreting which activity is related to local synaptic events, therefore, requires modeling of these multiple variables. Current-source density (CSD) analysis identifies the location and temporal dynamics of membrane current fluctuations and thus can serve to dissociate some of these variables (Leung, 1990). By electrically stimulating the tissue of interest from afferent regions, one can estimate the resistivity of the local extracellular space (near the recording electrode) through a simple ohmic relationship. This stimulation imposes current sources (where current flows into extracellular space) and sinks (current flows out of extracellular space) at recording sites such that the sum of membrane currents within a small volume (hence the "density") represents the CSD. The CSD profile, then, reflects extracellular current accumulation without volume conduction effects from nearby recording sites.

To make their measurements, Montgomery et al. (2009) implanted highdensity 96-site silicon probes in the dorsal hippocampus of behaving rats. The probes spanned almost the entire hippocampus in the dorsal-ventral axis and enabled the simultaneous recording of slow-wave signals and single-unit activity throughout the layers of regions CA1, dentate gyrus (DG), and CA3. This technique has been used before for studies of hippocampal and cortical laminar function (Montgomery and Buzsáki, 2007) but rarely for such a systematic survey of theta across hippocampal layers. In parallel to measuring the LFP, the authors also recorded local source and sink currents by CSD analysis. For both of these measures, they analyzed and compared power, frequency, coherence, and phase between different recording locations across the hippocampus under general conditions and when rats were either running on an elevated maze in a hippocampusdependent delayed spatial alternation task or in control tasks that were not dependent on the hippocampus (e.g., running back and forth on a track to get food reward).

Coherence of theta LFPs and currents were higher within a specific layer $(>0.98)$ than between layers $(>0.7)$ [Montgomery et al. (2009), their Fig. 1]. Analysis of phase differences of LFP theta, local theta currents, and single-unit activity between the different layers produced similar results: phase differences within a layer were much lower than differences between layers. The DG showed relatively high intralayer variability in both phase and coherence, likely attributable to the 
convergence of multiple cortical and intrahippocampal inputs [Montgomery et al. (2009), their Fig. $1 F]$ and their Fig. $2 D]$. These data suggest that each hippocampal layer is independent, to a certain degree, in terms of theta expression (as reflected by the LFP) and generation (as reflected by CSD analysis). These findings are also consistent with single-unit recording studies showing distinct coding properties of different hippocampal layers (Lee et al., 2004; Leutgeb et al., 2004) and extend these data to include slow-wave activity.

To assess the functional role of local theta activity, Montgomery et al. (2009) tested the influence of cognitive task demands on theta activity in the different hippocampal layers. Theta power, frequency, coherence, and phase were compared between layers as rats performed a hippocampus-dependent delayed spatial alteration task and hippocampusindependent control tasks. Employing a general linear model, the authors tested the relative influence of running speed, acceleration, and maze region on theta modulations (LFP and CSD) within and between layers. In the alteration task, different maze regions contributed substantially more to the variance of theta power between layers than different speeds and accelerations [Montgomery et al. (2009), their Fig. 4A-C]. Specifically, theta power in CA1 was selectively increased when rats were running in the central arm of the maze compared with other maze regions [Montgomery et al. (2009), their Fig. 4D, E]. Moreover, a significant increase in theta coherence in the DG was also observed in the central arm of the maze [Montgomery et al. (2009), their Fig. 6A-C]. Just like power and coherence, theta frequency increased when rats were running in the central arm, but this effect was more global and not restricted to a particular layer.

During hippocampus-independent tasks, the authors also report an increase in theta power in CA1 and theta coherence in the DG when rats occupied the initial physical sections of the running track [Montgomery et al. (2009), their Fig. 5 and their Fig. 6D]. Unlike the alternation task, theta frequency was not increased in any location of the control tasks. The layer-specific increase of theta power and theta coherence exclusively on the central arm may suggest that it is related to cognitive operations performed at this location. These may include memory retrieval (e.g., where did I go in the previous trial) and decision making (e.g., where should I go now). However, the fact that modulations of theta parameters were also observed in tasks that do not demand such cognitive operations (the control tasks) confounds the former interpretation. In contrast, the global increase in theta frequency only during the hippocampus-dependent alternation task may reflect faster information processing during periods of cognitive demand.

By combining LFP and CSD analysis, Montgomery et al. (2009) demonstrate that theta rhythmic activity in the hippocampus originates from multiple anatomically distributed current generators. What drives these current generators remains an open question. Hippocampal oscillations emerge from the recurrent organization of intrahippocampal networks, such as CA3, and from extrahippocampal structures, such as different layers of the entorhinal cortex and the medial septum. To investigate the multiple sources of theta oscillations, it will be necessary to record simultaneously from different locations within the hippocampus and in the entorhinal cortex, as well as other brain regions. Although voltagegated ion channels of hippocampal neurons have been implicated in local theta rhythms as well (Buzsáki, 2002), it is unclear whether they are required for behavior. Specific inhibition of voltage-gated channels in the context of behavior would clarify the role of these channels in information processing.

There is ample evidence that the theta phase of CA1 pyramidal cell spikes carries more information than firing rate alone (Huxter et al., 2008). The relative independence of both spiking and theta activity in other hippocampal layers, as shown by Montgomery et al. (2009), raises the question whether theta phase of spiking activity is equally informative in other hippocampal layers. Although the relationship between spikes and theta rhythm are clearly important in CA1, phase relations between theta and other types of slow waves may also be informative. The theta rhythm has been proposed to function as a carrier wave associated with gamma slow-wave activity $(30-50 \mathrm{~Hz})$. In a recent model of episodic memory encoding and retrieval proposed by Jensen and Lisman (2005), it was suggested that individual theta cycles "bind" together multiple components of a single memory; these "memory components" are represented by spikes on successive gamma rhythm cycles. Because it is likely that information processing is heterogeneous across the layers of the hippocampus, these findings together with previous re- ports on the layer and task specificity of hippocampal gamma rhythm (Montgomery and Buzsáki, 2007) suggest that gamma rhythmic activity may interact with theta in a local manner to guide behavior. If so, memory retrieval and encoding may be coordinated by cell assemblies whose axons and dendrites show layerdependent organization.

Montgomery et al. (2009) only partially support a role for layer-specific theta modulation in cognitive processes, mainly because similar modulations were observed during behaviors that were independent of the hippocampus. It is notable that hippocampal place fields, which are fundamental to hippocampal function, were discovered when animals were engaged in a hippocampal-independent task (O'Keefe and Dostrovsky, 1971). This is probably because hippocampal activity can be correlated with behavior even if it does not control it. With this in mind, the results of Montgomery et al. (2009), more than answering a particular question related to hippocampal function and cognitive operations, open a new perspective on the details of hippocampal function and call for further detailed investigations of the role of layer-specific theta in hippocampal information processing. One way of addressing this issue would be to increase cognitive demands imposed on the hippocampus while monitoring local theta activity; this can be achieved, for example, by letting the animal perform the familiar hippocampus-dependent task in a novel room. Successful performance in the novel environment would require the flexible association of novel and familiar information, the kind of operations suggested to depend on the hippocampus (Eichenbaum, 2004).

Although the functions of theta oscillations have been long debated, there is still no clear consensus on what information processing roles they serve in the hippocampus. Proceeding from the assumption that theta rhythm is not a monolithic phenomenon, we may now be able to ask better questions about its function in processes of learning and memory.

\section{References}

Bland BH, Oddie SD (2001) Theta band oscillation and synchrony in the hippocampal formation and associated structures: the case for its role in sensorimotor integration. Behav Brain Res 127:119-136.

Buzsáki G (2002) Theta oscillations in the hippocampus. Neuron 33:325-340.

Eichenbaum H (2004) Hippocampus: cognitive 
processes and neural representations that underlie declarative memory. Neuron 44:109-120.

Huxter JR, Senior TJ, Allen K, Csicsvari J (2008) Theta phase-specific codes for two-dimensional position, trajectory and heading in the hippocampus. Nat Neurosci 11:587-594.

Jensen O, Lisman JE (2005) Hippocampal sequence-encoding driven by a cortical multiitem working memory buffer. Trends Neurosci 28:67-72.

Kahana MJ (2006) The cognitive correlates of human brain oscillations. J Neurosci 26:1669-1672.
Lee I, Rao G, Knierim JJ (2004) A double dissociation between hippocampal subfields: differential time course of CA3 and CA1 place cells for processing changed environments. Neuron 42:803-815.

Leung LS (1990) Field potentials in the central nervous system. In: Neuromethods: neurophysiological techniques (Boulton AA, Baker GB, Vanderwolf CH, eds), pp 277-312. Totowa, NJ: Humana.

Leutgeb S, Leutgeb JK, Treves A, Moser MB, Moser EI (2004) Distinct ensemble codes in hippocampal areas CA3 and CA1. Science 305:1295-1298.
Montgomery SM, Buzsáki G (2007) Gamma oscillations dynamically couple hippocampal $\mathrm{CA} 3$ and CA1 regions during memory task performance. Proc Natl Acad Sci USA 104:14495-14500.

Montgomery SM, Betancur MI, Buzsáki G (2009) Behavior-dependent coordination of multiple theta dipoles in the hippocampus. J Neurosci 29:1381-1394.

O’Keefe J, Dostrovsky J (1971) The hippocampus as a spatial map. preliminary evidence from unit activity in the freely-moving rat. Brain Res 34:171-175. 